\title{
Prevalence of Metabolic Syndrome in the city of São Paulo, Brazil, an observational study
}

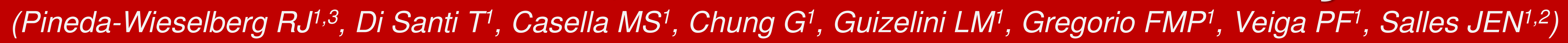

1. Faculdade de Ciências Médicas da Santa Casa de São Paulo (FCMSCSP), Liga Acadêmica de Endocrinologia e Metabologia

2. Sociedade Brasileira de Diabetes

3. International Diabetes Federation - Young Leader in Diabetes Programme

Contact:

\section{Introduction}

Metabolic syndrome (MS) implies in several metabolic dysfunction and it is related to chronic diseases, being an important risk factor for cardiovascular events. Its prevalence has been growing around the world; studies in developing countries points a varying prevalence between $12.3 \%$ and $42.7 \%$, depending on the utilized criteria. It is important to know MS prevalence for effective prevention and therapeutic approach. Our target is to analyze the prevalence of MS in the local population in a period of three years.

\section{Methods}

The data were collected between the years 2015 and 2017 in health events in downtown São Paulo, Brazil. In such events, medical students measured people's arterial blood pressure (BP), body mass index (BMI), waist circumference (WC) and capillary blood glucose (BG), and collected others informations. These people came by free will, and after the data was collected, were oriented about weight adequacy, regular physical activities practices, selfmedication, and screening for noncommunicable diseases (NCD). A MD supervised all activities. The data was compared with the IDF diagnosis criteria: central obesity (WC $\geq 94 \mathrm{~cm}$ for men and $\geq 80 \mathrm{~cm}$ for women or BMI >30) and two or more of the following: specific treatment for dyslipidemia (DLP); for high blood pressure (HBP); or for diabetes mellitus (DM).

\section{Results}

In three years, we obtained 838 entries $(\mathrm{Cl}=95 \%)$, of those, 445 were women and 393 were men. The average prevalence of MS in these three years was $12.26 \%$ for women and $8.93 \%$ for men (10.59\% for both genders). Detailed results can be observed in Graphs 1 to 4 .

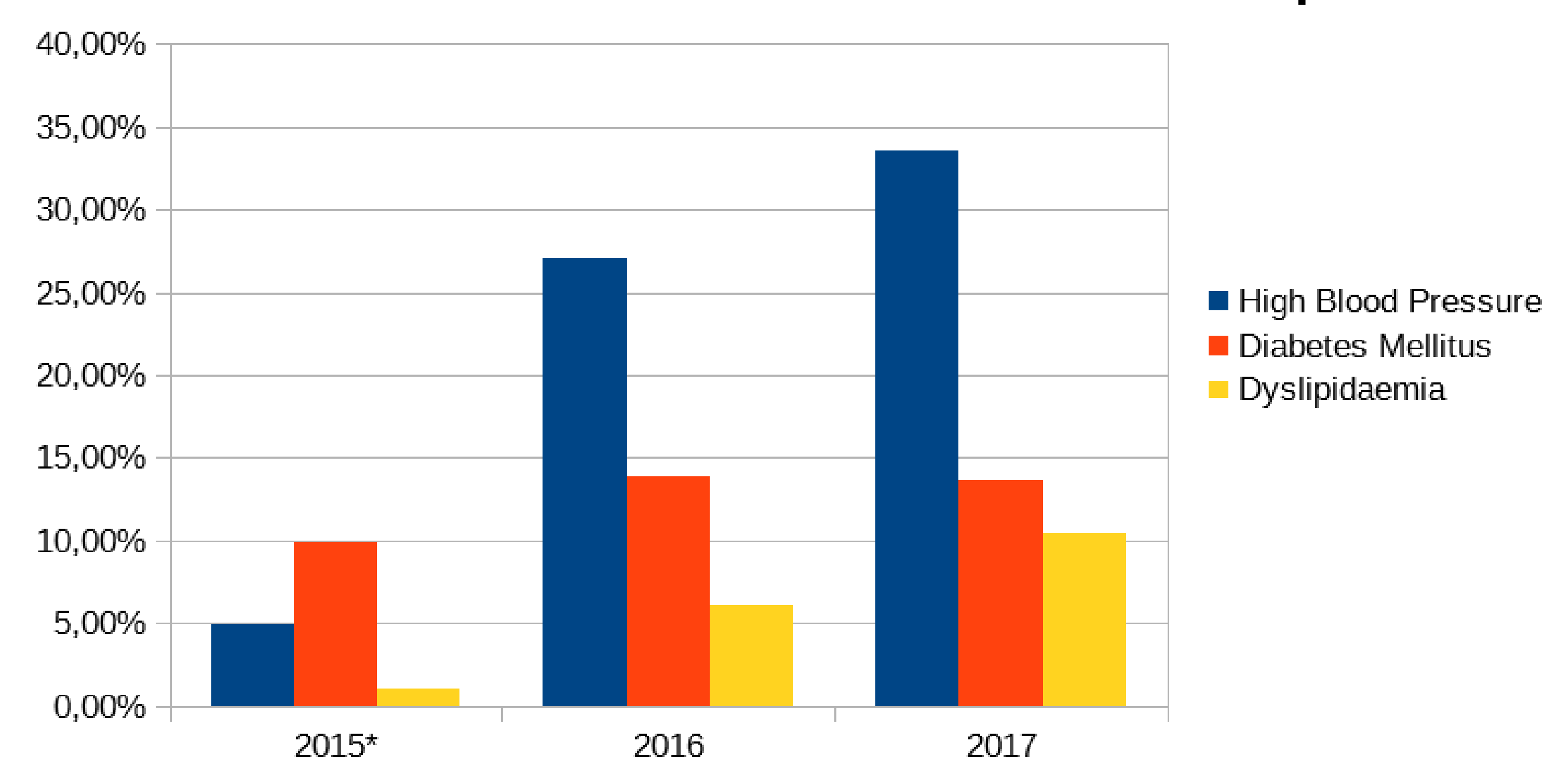

Graph 1: Percentage of men with high blood pressure, Diabetes Mellitus and Dyslipidaemia, 2015 through 2017

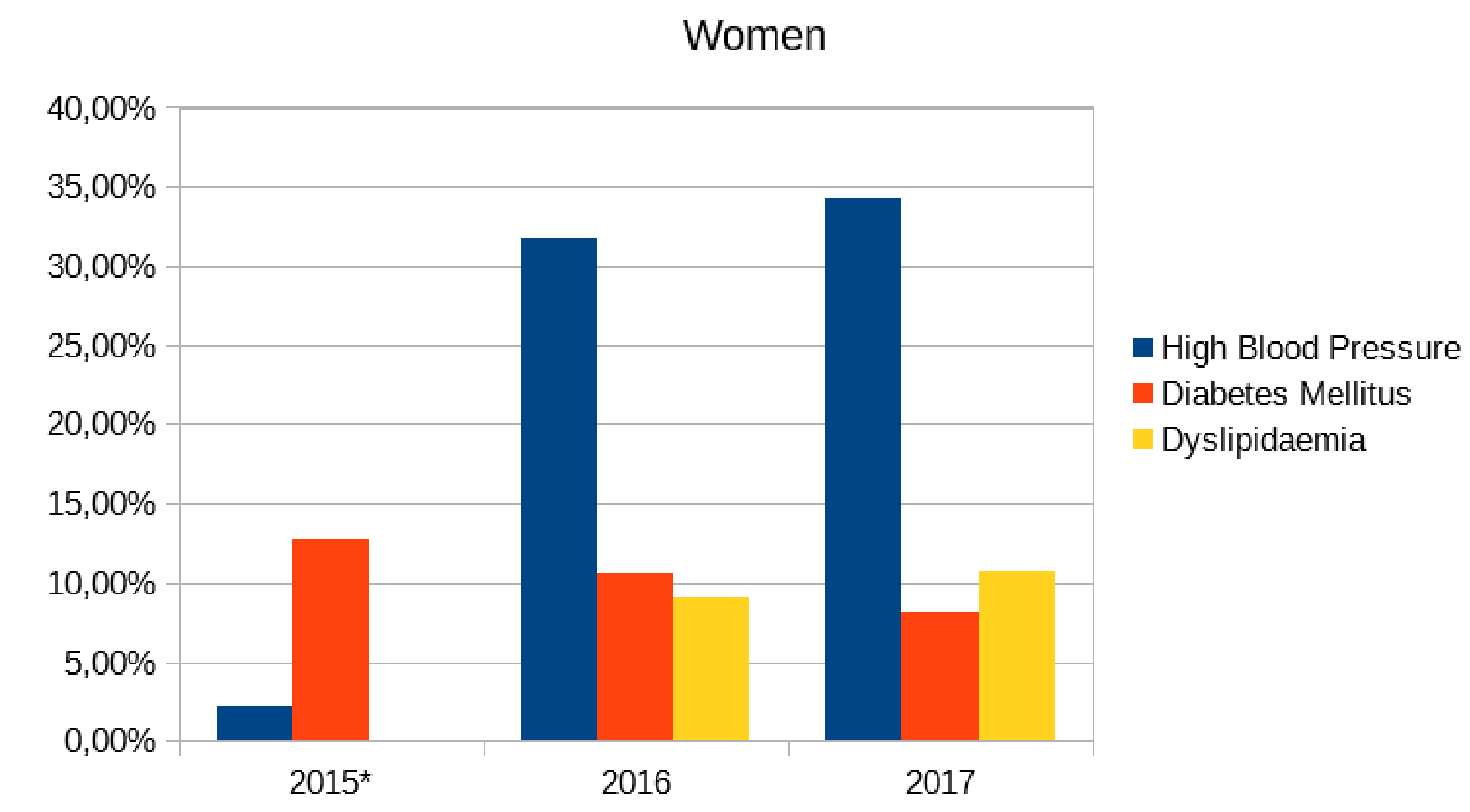

Graph 2: Percentage of women with high blood pressure, Diabetes Mellitus and Dyslipidaemia, 2015 through 2017

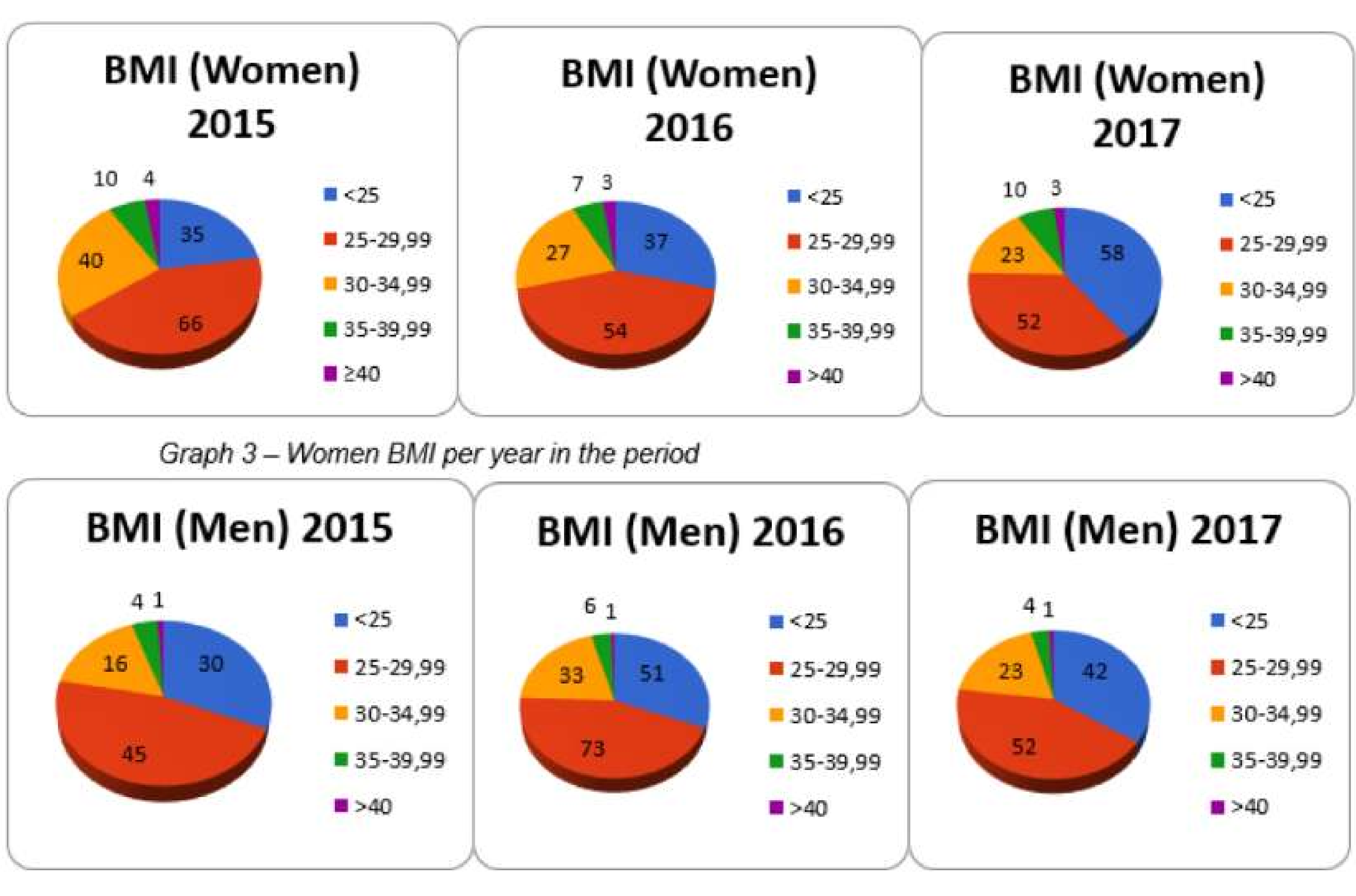

Graphic 4 - Men BMI per year in the period

\section{Discussion}

The results indicate a satisfactory representation of São Paulo's population. The average WC and BMI above recommended points toward a deficiency in the basic healthcare system. The variation of the data in regards the prevalence of HBP, DM and DLP indicates that the healthcare professionals gave variable importance to NCD rising concerns about medical education and professional preparation. There is a possibility that the patients did not know how to inform their NCD, and once the responsible one for the patients' instruction is the MD, the former concern cannot be dismissed. Even with the variation of the collected data, MS prevalence was high and, therefore, the subject must be approached more frequently, as new strategies to control and screen this condition must be developed.

\section{References}

1. International Diabetes Federation. Information on the IDF consensus worldwide definition of the metabolic syndrome, 2010.

2. Moreire GC, Prevalence of Metabolic Syndrome: Association with Risk Factors and Cardiovascular Complications in an Urban Population, State Medical School in São José do Rio Preto (FAMERP), 2014. 